\title{
Injection of absolute alcohol into cysts cavities, after cyst aspiration, for treating and reducing the rate of recurrence of benign breast cysts
}

\author{
Dagoberto Álvarez-Fernández • José L. González • \\ Juan Carlos Ayala-Luna • Martha Rodríguez-Morán • \\ Fernando Guerrero-Romero
}

Received: 3 September 2009 /Accepted: 15 January 2010/Published online: 13 February 2010

(C) Springer-Verlag 2010

\begin{abstract}
The purpose of this study is to determine whether injection of absolute alcohol into cysts cavities, after cyst aspiration, is useful to treat benign breast cysts (BBCs) and to reduce its rate of recurrence. Fifteen women with recurrent $\mathrm{BBC}$ were enrolled in a 3.5 years follow-up study. Cyst aspiration and replacement by an equal volume of absolute alcohol (99\%) within cysts cavities was performed under guidance of ultrasonography. BBC was defined as a breast cyst without cytologic changes for malignancy. Ultrasonographic breast images and breast biopsies were performed to verify cyst resolution and recurrence. Breast cysts completely disappeared in six $(40 \%)$ women and significantly decreased its average size ( $67 \pm 38$ to $5.2 \pm 6.8 \mathrm{~mm}, p=0.002$ ) in eight $(53.3 \%$ ) women. At the end of the follow-up (42.1 \pm 13.3 months) study, 14
\end{abstract}

D. Álvarez-Fernández

Surgical Oncology Department,

General Hospital of the Mexican Social Security Institute, Durango, Mexico

J. L. González

Pathology Department,

General Hospital of the Mexican Social Security Institute, Durango, Mexico

J. C. Ayala-Luna

Gynecology Department,

General Hospital of the Mexican Social Security Institute, Durango, Mexico

M. Rodríguez-Morán · F. Guerrero-Romero $(\bowtie)$

Biomedical Research Unit,

General Hospital of the Mexican Social Security Institute,

Siqueiros 225 esq./Castañeda,

34000 Durango, Durango, Mexico

e-mail: guerrero_romero@hotmail.com women $(93.3 \% ; 14 / 15)$ were free of recurrence; one $(6.7 \%)$ woman did not show variation $(54 \mathrm{~mm})$ in the size of BBC. Alcohol sclerotherapy can then be considered as a useful alternative option in the treatment of recurrent BBC.

Keywords Benign breast cysts · Alcohol · Sclerotherapy

\section{Introduction}

Benign lesions of the breast are more frequent than malignant ones [1-4]. Usually, breast sonography is adequate to accomplish the diagnoses of benign breast disease; however, in special cases the use of mammography, ultrasound, magnetic resonance, and needle biopsies, are needed to achieve diagnosis [5]. However, the major concern is about the management of benign breast cysts; on one hand, the accepted standard practice is the surveillance and follow-up with imaging studies instead of intervention [6] but, on the other hand, it has been reported that intervention for diagnosis and treatment such as the sonographically guided vacuum-assisted biopsy and pneumocystography reduces the recurrence rate of benign breast cysts [7-9]. Given that majority of benign lesions are not associated with an increased risk of breast cancer, unnecessary surgical procedures should be avoided [5]; however, because women with breast cysts commonly suffer of pain and anxiety, nonsurgical alternative procedures, accessible in the clinical setting for treating benign breast cysts, are needed. On this regard, the objective of this study was to determine whether the injection of absolute alcohol into cysts cavities, after cyst aspiration, is useful to treat benign breast cysts and to reduce its rate of recurrence. 


\section{Materials and methods}

A total of 15 women with benign recurrent breast cysts and history of treatment failure using needle aspiration of breast cysts, were enrolled in a 3.5 years follow-up study carriedout in the General Hospital of the Mexican Social Security Institute in Durango, Mexico. The protocol was approved by the Institutional Ethic Board. Before intervention, all women gave their informed consent.

Ultrasonographic breast image and fine-needle aspiration of breast cysts were performed for evaluating malignancy. Clinical or ultrasonographic signs suggesting malignancy and the presence of malignant cells in the cytologic examination were exclusion criteria.

Cyst aspiration and alcohol injection within cysts cavities were performed under the guidance of ultrasonography. A 22-gauge needle was passed into the cysts, and fluid was aspirated; subsequently, an equal volume of absolute alcohol (99\%; Delta Laboratory diagnostic, Mexico D.F.) was injected into the cyst cavity.

Ultrasonographic breast images were scheduled every 4 months after alcohol injection to verify cyst recurrence. In nine $(60 \%)$ women who had multiple breast cysts, all cysts were treated with alcohol injection into cysts cavities. Additionally, in these nine women, an excisional biopsy was performed on one breast cyst in order to evaluate the histological changes secondary to the alcohol therapy; only one biopsy per woman was performed. The biopsies were achieved in days 14, 30, 45, 75, 95 and 105.

Excisional breast biopsy on one cyst was performed to evaluate histologic changes induced by injection of absolute alcohol.

Statistical analysis The primary trial end point of this pilot study was to evaluate the reduction in the rate of recurrence of breast cysts. The preplanned intention-to-treat analysis of the primary study end point was done for all the participants. Differences were assessed by two-tailed paired Student's $t$ test for comparison of normally distributed variables (Mann-Whitney $U$ test for skewed data) or chisquare test (Fisher's exact test) for categoric variables.

\section{Results}

There were no adverse events or side effects due to injection of absolute alcohol in the cyst cavity. Women did not complain of pain, irritation or inconvenience in the site of injection; edema did not develop in the subsequent days.

The average $\pm \mathrm{SD}$ of age and follow-up was $45.6 \pm$ 7.6 years and $42.1 \pm 13.3$ months (range, 15 to 55 months), respectively. Table 1 shows the characteristics of the women enrolled. Menopause was identified in $40.0 \%$ of the women. None of the women received oral anticontraceptive therapy or hormonal treatment.

Of the 15 punctured cysts, 12 (80\%) had complete, and three $(20 \%)$ partial substitution of the cyst fluid by absolute alcohol. At end of follow-up period, breast cysts in six (40\%) women completely disappeared and eight $(53.3 \%)$ significantly decreased its average size $(67 \pm 38$ to $5.2 \pm 6.8 \mathrm{~mm}, p=0.002)$; all these women $(93.3 \% ; 14 /$ $15)$ were free of recurrence. Only one $(6.7 \%)$ woman did not show any variation $(54 \mathrm{~mm})$ in the size of the breast cyst.

Among women in whom the breast cyst completely disappeared, four $(66.6 \%)$ were premenopausal. The woman who showed no response was premenopausal, and there was partial substitution of cyst fluid by absolute alcohol, Table 1.

Injection of absolute alcohol in the breast cyst cavity induced a local inflammatory reaction and fibrosis that promotes cyst scar. The histologic changes induced by injection of absolute alcohol are scarce, subtle, and unspecific. At 14 days post-injection of absolute alcohol, there were interstitial bleeding and unspecific panniculitis on the estrome of fibroadipose connective tissue as well as necrosis and sparkling macrophages on the adipose tissue. At 30 days, there were small cysts cavities, with either flattened cubic epithelium or without epithelium. At 73 days, the small cysts cavities remain present, these cysts are distorted, collapsed, and without epithelium; in

Table 1 Menopausal status of women enrolled in the study

\begin{tabular}{lllll}
\hline Patient & Age & Menopause & $\begin{array}{l}\text { Use of hormonal } \\
\text { therapy and/or oral } \\
\text { anticontraceptive pill }\end{array}$ & $\begin{array}{l}\text { Cyst } \\
\text { resolution } \\
(\%)\end{array}$ \\
\hline 1 & 34 & No & No & 100 \\
2 & 43 & No & No & 100 \\
3 & 61 & Yes & No & 100 \\
4 & 61 & Yes & No & 100 \\
5 & 36 & No & No & 100 \\
6 & 42 & No & No & 100 \\
7 & 48 & Yes & No & 96 \\
8 & 45 & No & No & 94 \\
9 & 50 & Yes & No & 33 \\
10 & 43 & No & No & 98 \\
11 & 41 & No & No & 56 \\
12 & 45 & Yes & No & 88 \\
13 & 43 & No & No & 81 \\
14 & 50 & Yes & No & 86 \\
15 & 42 & No & No & 0 \\
\hline
\end{tabular}


addition, periductal fibrosis of the connective tissue was also observed. At 95 days, resolution of inflammatory process and lax connective tissue, substituting the adipose tissue, were the histologic features. Finally, at day 105, clefts and collapsed spaces, with flattened epithelium or absence of epithelium were observed, Fig. 1.

\section{Discussion}

Results of this study show that replacing breast cyst fluid with an equal volume of absolute alcohol, induce collapse and fibrosis of the benign breast cyst cavities and reduce its recurrence. Our results suggest that alcohol sclerotherapy could be a useful procedure in the treatment of benign breast cyst; the procedure produces minimum disturbances and it has no adverse events.

Fine-needle aspiration under sonographic guidance is a safe and common therapeutic procedure of benign breast cyst; however, because cyst persistence or recurrence is frequent, methods such as the pneumocystography have been proposed to increase the successful outcomes [7, 9]. After cyst aspiration, the injection of air in the cyst cavity produces collapse of the cyst and reduces its recurrence [9]. In a similar way, our results show that injection of absolute alcohol into the empty breast cyst cavity produces its collapse and induces a local inflammatory reaction and fibrosis that promotes cyst scar, with the consequent reduction of the cyst recurrence.

Ikeda et al. [7], who reviewed hospital records and mammograms of 38 women with 41 impalpable breast cysts, reported that the pneumocystography procedure was successful in the treatment of $18(43.9 \%)$ and unsuccessful in 20 of the 41 cysts. In the follow-up (range, 4 months to 3 years), they found that three (17\%) of 18 cysts in the group with successful pneumocystography recurred. In the present study, injection of absolute alcohol to replace the volume of aspirated cyst fluid was successful in $80 \%$ of the procedures and the rate of recurrence was $6.7 \%$. However, rather than a preferable method, the injection of absolute alcohol should be placed as an alternative procedure should pneumocystography fail. However, it is necessary to keep in mind that more than half of the women with documented evidence of interval benign breast cysts show spontaneous regression of the lesion in 1 year, most of them completely, and by 2 years, nearly two thirds regressed without intervention [10].

Techniques of alcohol sclerotherapy for the treatment of large Hepatic cysts has been extensively used, showing that long retention of alcohol in a single injection is safe and effective [11], suggesting that alcohol sclerotherapy can be performed safely and efficaciously. To the best of our knowledge, this is the first report regarding the use of
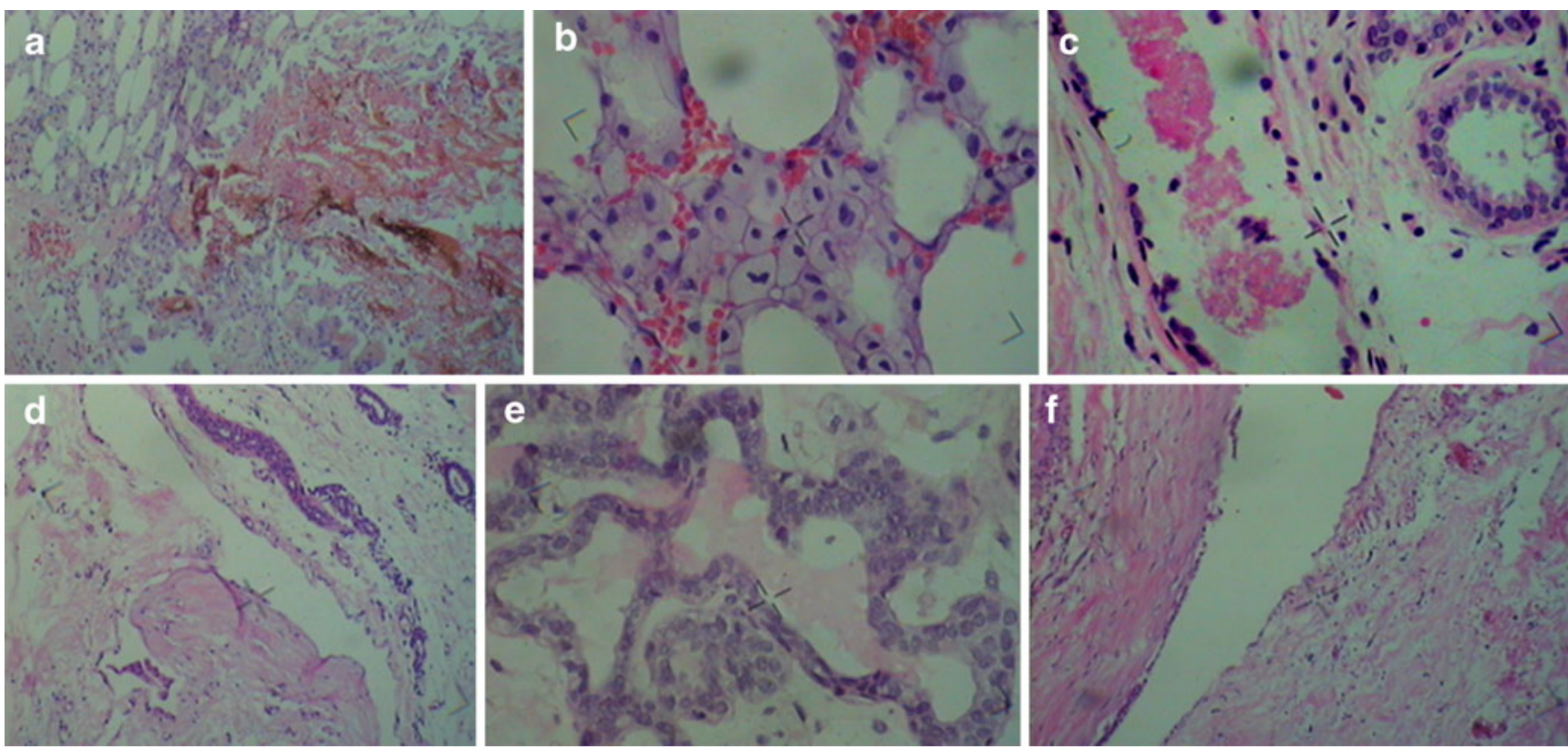

Fig. 1 Progressive histological changes from day 14 to 105 after replace the breast cyst fluid by an equal volume of absolute alcohol. a Fibrinoid necrosis, interstitial bleeding and paniculitis. b Interstitial bleeding and foam macrophages. $\mathbf{c}$ Intracystic fibrinoid material and coating epithelium with degenerative process. $\mathbf{d}$ Collapsed breast cyst cavity with residual epithelium on degenerative process 1 month after injection of alcohol. e Fibrocystic mastopathy with coating epithelium and prominent cubic cells. f Collapsed cyst cavity without epithelium 105 days after injection of alcohol 
alcohol sclerotherapy for the treatment of benign breast cysts.

Some limitations of the study deserve to be mentioned. Firstly, the absence of a control group to appropriately demonstrate the efficacy of the injection of absolute alcohol for treating benign breast cysts; however, because this is a case report study, the control group was not necessary. Further research based on randomized equivalent clinical trials is required to compare the efficacy of the injection of absolute alcohol into cysts cavities, after cyst aspiration, versus the usual procedures to treat benign breast cysts. Secondly, it should be taken into account that a great majority of the benign breast cysts interval shows spontaneous regression that might affect our findings. In upcoming research in this field, meticulous evaluation of benign breast cysts will be required before intervention in order to control this potential confounder variable.

In conclusion, our data show that after cyst aspiration, replacing cyst liquid with an equal amount of absolute alcohol reduce cysts recurrence. Alcohol sclerotherapy can be a useful procedure in the treatment of benign breast cyst.

Acknowledgements This work was partially supported by grants from the Mexican Social Security Institute Foundation, Civil Association.

Conflict of interest There is no actual or potential conflict of interest in relation to this article.

\section{References}

1. Caleffi M, Filho DD, Borghetti K, Graudenz M, Littrup PJ, Freeman-Gibb LA, Zannis VJ, Schultz MJ, Kaufman CS, Francescatti D, Smith JS, Simmons R, Bailey L, Henry CA, Stocks LH (2004) Cryoablation of benign breast tumors: evolution of technique and technology. Breast 13:397-407

2. Cole P, Mark Elwood J, Kaplan SD (1978) Incidence rates and risk factors of benign breast neoplasm. Am J Epidemiol 108:112-120

3. Sarnelli R, Squartini F (1991) Fibrocystic condition and "at risk" lesions in asymptomatic breasts: a morphologic study of postmenopausal women. Clin Exp Obstet Gynecol 18:271-279

4. Cook MG, Rohan TE (1985) The patho-epidemiology of benign proliferative epithelial disorders of the female breast. J Pathol 146:1-15

5. Venta LA, Kim JP, Pelloski CE, Morrow M (1999) Management of complex breast cysts. AJR Am J Roentgenol 173:1331-1336

6. O'Malley FP, Bane AL (2004) The spectrum of apocrine lesions of the breast. Adv Anat Pathol 11:1-9

7. Ikeda DM, Helvie MA, Adler DD, Schwindt LA, Chang AE, Rebner M (1992) The role of fine-needle aspiration and pneumocystography in the treatment of impalpable breast cysts. AJR Am J Roentgenol 158:1239-1241

8. Ko ES, Han H, Lee BH, du Choe H (2009) Sonographic changes after removing all benign breast masses with sonographically guided vacuum-assisted biopsy. Acta Radiol 50(9):968-974

9. Tabár L, Péntek Z, Dean PB (1981) The diagnostic and therapeutic value of breast cyst puncture and pneumocystography. Radiology 141:659-663

10. Brenner RJ, Bein ME, Sarti DA, Vinstein AL (1994) Spontaneous regression of interval benign cysts of the breast. Radiology 193:365-368

11. Yang Ch-F, Liang H-L, Pan H-B, Lin Y-H, Mok K-T, Lo G-H, Lai K-H (2006) Single-session prolonged alcohol-retention sclerotherapy for large hepatic cysts. AJR Am J Roentgenol 187:940-943 